\title{
BOUNDEDNESS OF ROUGH SINGULAR INTEGRAL OPERATORS ON HOMOGENEOUS HERZ SPACES
}

\author{
GUOEN HU, SHANZHEN LU and DACHUN YANG
}

(Received 5 January 1998; revised 9 October 1998)

\author{
Communicated by A. H. Dooley
}

\begin{abstract}
The authors establish the boundedness on the Herz spaces and the weak Herz spaces for a large class of rough singular integral operators and their corresponding fractional versions. Applications are given to Fefferman's rough singular integral operators, their fractional versions, their commutators with $\mathrm{BMO}\left(\mathbb{R}^{n}\right)$ functions and Ricci-Stein oscillatory singular integral operators. Some new results are obtained.

1991 Mathematics subject classification (Amer. Math. Soc.): primary 42B20; secondary 42B25, 47A30, 47B38.

Keywords and phrases: Herz space, Lebesgue space, power weight, singular integral, fractional singular integral.
\end{abstract}

\section{Introduction}

A well-known result of Stein [23] states that if

$$
T f(x)=\text { p.v. } \int_{\mathbb{R}^{n}} H(x-y) f(y) d y
$$

is bounded on $L^{q}\left(\mathbb{R}^{n}\right)$ for some $q \in(1, \infty)$, and if the kernel $H$ satisfies the 'standard size' condition

$$
|H(x)| \leq \frac{c}{|x|^{n}} \text { for all } x \in \mathbb{R}^{n} \backslash\{0\}
$$

then $T$ is also bounded on $L_{|x|^{\alpha}}^{q}\left(\mathbb{R}^{n}\right)$ for $\alpha \in(-n, n(q-1))$. Here, and in what follows, $f \in L_{\omega}^{q}\left(\mathbb{R}^{n}\right)$ for any non-negative function $\omega$ on $\mathbb{R}^{n}$ means that $f$ is a measurable

The research was partially supported by the SEDF and the NNSF of China.

(C) 1999 Australian Mathematical Society 0263-6115/99 $\$ A 2.00+0.00$ 
function and

$$
\|f\|_{L_{\omega}^{q}\left(\mathbb{R}^{n}\right)}=\left(\int_{\mathbb{R}^{n}}|f(x)|^{q} \omega(x) d x\right)^{1 / q}<\infty .
$$

Let $\Omega$ be a homogeneous function of degree zero on $\mathbb{R}^{n}$ and $\Omega \in L^{r}\left(S^{n-1}\right)$ for some $r \in[1, \infty]$. If $T$ in $(1.1)$ is bounded on $L^{q}\left(\mathbb{R}^{n}\right)$ for some $q \in(1, \infty)$, and $H$ satisfies, instead of (1.2),

$$
|H(x)| \leq c \frac{|\Omega(x)|}{|x|^{n}} \text { for all } x \in \mathbb{R}^{n} \backslash\{0\}
$$

what then can be said on the boundedness of $T$ on $L_{|x|^{\alpha}}^{q}\left(\mathbb{R}^{n}\right)$ ? The main purpose of this paper is to answer this question. Note that the homogeneous Herz spaces are some kind of generalizations of the weighted Lebesgue spaces. We establish our results in the setting of the Herz spaces.

Let $B_{k}=B\left(0,2^{k}\right)=\left\{x \in \mathbb{R}^{n}:|x| \leq 2^{k}\right\}$ and $A_{k}=B_{k} \backslash B_{k-1}$ for any $k \in \mathbb{Z}$. Denote $\chi_{A_{k}}$ by $\chi_{k}$ for any $k \in \mathbb{Z}$, where $\chi_{E}$ is the characteristic function of the set $E$.

DefinITION 1.1. Let $\alpha \in \mathbb{R}, 0<p \leq \infty$ and $0<q \leq \infty$. The homogeneous Herz spaces $\dot{K}_{q}^{\alpha, p}\left(\mathbb{R}^{n}\right)$ are defined by

$$
\dot{K}_{q}^{\alpha, p}\left(\mathbb{R}^{n}\right)=\left\{f \in L_{l o c}^{q}\left(\mathbb{R}^{n} \backslash\{0\}\right):\|f\|_{K_{q}^{\alpha . p}\left(\mathbb{R}^{n}\right)}<\infty\right\},
$$

where

$$
\|f\|_{\dot{K}_{q}^{\alpha, p}\left(\mathbb{R}^{n}\right)}=\left\{\sum_{k=-\infty}^{\infty} 2^{k \alpha p}\left\|f \chi_{k}\right\|_{L^{q}\left(\mathbb{R}^{n}\right)}^{p}\right\}^{1 / p},
$$

and the usual modifications are made when $p=\infty$ or $q=\infty$.

Obviously, $\dot{K}_{q}^{0 . q}\left(\mathbb{R}^{n}\right)=L^{q}\left(\mathbb{R}^{n}\right)$ and $\dot{K}_{q}^{\alpha / q, q}\left(\mathbb{R}^{n}\right)=L_{|x|^{q}}^{q}\left(\mathbb{R}^{n}\right)$. We remark that studies involving these spaces have a long history; and we recommend the references $[9,14,17]$ and $[12]$.

Soria and Weiss [22] give some beautiful generalizations of Stein's results in [23]. In particular, they obtain the result of Stein in the case $q=1$. We generalize the results of Soria and Weiss in the case $q=1$ to weak Herz spaces which are introduced by the authors in [12].

In what follows, for any $k \in \mathbb{Z}$ and any $\sigma>0$, let $m_{k}(\sigma, f)=\mid\left\{x \in A_{k}:|f(x)|>\right.$ $\sigma\} \mid$. 
DEFINITION 1.2. ([12]) Let $\alpha \in \mathbb{R}, 0<q<\infty$ and $0<p \leq \infty$. A measurable function $f$ on $\mathbb{R}^{n}$ is said to belong to the homogeneous weak Herz space $W \dot{K}_{q}^{\alpha, p}\left(\mathbb{R}^{n}\right)$, if

$$
\|f\|_{W \dot{K}_{q}^{\alpha, p}\left(\mathbb{R}^{n}\right)}=\sup _{\lambda>0} \lambda\left\{\sum_{k=-\infty}^{\infty} 2^{k \alpha p} m_{k}(\lambda, f)^{p / q}\right\}^{1 / p}<\infty,
$$

where the usual modifications are made when $p=\infty$.

Clearly, $W \dot{K}_{q}^{0, q}\left(\mathbb{R}^{n}\right)=W L^{q}\left(\mathbb{R}^{n}\right)$ and $W \dot{K}_{q}^{\alpha / q, q}\left(\mathbb{R}^{n}\right)=W L_{|x|^{q}}^{q}\left(\mathbb{R}^{n}\right)$. Here $f \in$ $W L_{\omega}^{q}\left(\mathbb{R}^{n}\right)$ means that $f$ is a measurable function on $\mathbb{R}^{n}$ and

$$
\sup _{\lambda>0} \lambda\left[\omega\left(\left\{x \in \mathbb{R}^{n}:|f(x)|>\lambda\right\}\right)\right]^{1 / q} \leq c<\infty .
$$

The smallest constant c satisfying (1.4) is denoted by $\|f\|_{w L_{w}^{q}\left(\mathbb{R}^{n}\right)}$.

Let $0<l<n$. Instead of (1.3), if $H$ satisfies

$$
|H(x)| \leq c \frac{|\Omega(x)|}{|x|^{n-l}} \text { for all } x \in \mathbb{R}^{n} \backslash\{0\},
$$

what will happen for the operator $T$ defined by (1.1)? We obtain some similar results to that operator $T$ with a kernel $H$ satisfying (1.5).

Our main theorems are stated in Section 2; and their proofs are also given in this section. In Section 3, we give some applications. Based on the results of Fan-Pan [6], Seeger [21], Ding [4], Chanillo-Christ [1], Christ-Rubio de Francia [2], Jiang-Lu [13] and Pan [19], we obtain some new results on rough singular integrals, fractional rough singular integrals and oscillatory singular integral operators.

We should point out that some special cases of our theorems have been obtained in $[16,10]$, and [15]. Some of the techniques used here are borrowed from Soria-Weiss [22]; see also [14].

Throughout this paper, $c$ always means a constant independent of the main parameters involved, but may be different from line to line. For any power exponent $p$ with $1 \leq p \leq \infty$, we denote the conjugate exponent $p /(p-1)$ by $p^{\prime}$.

\section{Main theorems and their proofs}

To begin with, the following theorem indicates that the boundedness of operator $T$ on Lebesgue spaces with power weights implies its boundedness on homogeneous Herz spaces.

THEOREM 2.1. If a sublinearoperator $T$ is bounded on $L_{|x|^{\beta}}^{q}\left(\mathbb{R}^{n}\right)$ for all $\beta \in\left(\beta_{1}, \beta_{2}\right)$ and some $q \in(1, \infty)$, where $\beta_{1}, \beta_{2} \in \mathbb{R}$, then $T$ is also bounded on $\dot{K}_{q}^{\alpha, p}\left(\mathbb{R}^{n}\right)$ provided that $\alpha \in\left(\beta_{1} / q, \beta_{2} / q\right)$ and $0<p \leq \infty$. 
PROOF. We choose $\alpha_{1}, \alpha_{2} \in \mathbb{R}$ such that $\beta_{1} / q<\alpha_{1} / q<\alpha<\alpha_{2} / q<\beta_{2} / q$. Write

$$
f(x)=\sum_{k=-\infty}^{\infty} f(x) \chi_{k}(x) \equiv \sum_{k=-\infty}^{\infty} f_{k}(x)
$$

By the condition of the theorem, we then have

$$
\begin{aligned}
& \|T f\|_{\dot{K}_{q}^{a, p}\left(\mathbb{R}^{n}\right)}=\left\{\sum_{k=-\infty}^{\infty} 2^{k \alpha p}\left\|\chi_{k} T f\right\|_{L^{q}\left(\mathbb{R}^{n}\right)}^{p}\right\}^{1 / p} \\
& \leq c\left\{\sum_{k=-\infty}^{\infty} 2^{k \alpha p}\left(\sum_{j=-\infty}^{k+1}\left\|\chi_{k} T f_{j}\right\|_{L^{q}\left(\mathbf{R}^{n}\right)}\right)^{p}\right\}^{1 / p} \\
& +c\left\{\sum_{k=-\infty}^{\infty} 2^{k \alpha p}\left(\sum_{j=k+2}^{\infty}\left\|x_{k} T f_{j}\right\|_{L^{q}\left(\mathbf{R}^{n}\right)}\right)^{p}\right\}^{1 / p} \\
& \leq c\left\{\sum_{k=-\infty}^{\infty} 2^{k\left(\alpha-\alpha_{2} / q\right) p}\left(\sum_{j=-\infty}^{k+1}\left\|\chi_{k} T f_{j}\right\|_{L_{|x|^{\alpha_{2}}}^{q}\left(\mathbb{R}^{n}\right)}\right)^{p}\right\}^{1 / p} \\
& +c\left\{\sum_{k=-\infty}^{\infty} 2^{k\left(\alpha-\alpha_{1} / q\right) p}\left(\sum_{j=k+2}^{\infty}\left\|x_{k} T f_{j}\right\|_{L_{\left.k j\right|^{\alpha}}^{q}\left(\mathbf{R}^{n}\right)}\right)^{p}\right\}^{1 / p} \\
& \leq c\left\{\sum_{k=-\infty}^{\infty} 2^{k\left(\alpha-\alpha_{2} / q\right) p}\left(\sum_{j=-\infty}^{k+1}\left\|f_{j}\right\|_{L_{|x|^{\alpha_{2}}\left(\mathbb{R}^{n}\right)}}\right)^{p}\right\}^{1 / p} \\
& +c\left\{\sum_{k=-\infty}^{\infty} 2^{k\left(\alpha-\alpha_{1} / q\right) p}\left(\sum_{j=k+2}^{\infty}\left\|f_{j}\right\|_{\left.L_{|x|}^{q}\right|_{\mid} ^{q}\left(\mathbb{R}^{n}\right)}\right)^{p}\right\}^{1 / p} \\
& \leq c\left\{\sum_{k=-\infty}^{\infty}\left(\sum_{j=-\infty}^{k+1} 2^{j \alpha}\left\|f_{j}\right\|_{L^{q}\left(\mathbb{R}^{n}\right)} 2^{(k-j)\left(\alpha-\alpha_{2} / q\right)}\right)^{p}\right\}^{1 / p} \\
& +c\left\{\sum_{k=-\infty}^{\infty}\left(\sum_{j=k+2}^{\infty} 2^{j \alpha}\left\|f_{j}\right\|_{L^{q}\left(\mathbb{R}^{n}\right)} 2^{(k-j)\left(\alpha-\alpha_{1} / q\right)}\right)^{p}\right\}^{1 / p} .
\end{aligned}
$$

If $0<p \leq 1$, the above quantity is dominated by

$$
\begin{aligned}
c\left\{\sum_{k=-\infty}^{\infty}\right. & \left.\left(\sum_{j=-\infty}^{k+1} 2^{j \alpha p}\left\|f_{j}\right\|_{L^{q}\left(\mathbb{R}^{n}\right)}^{p} 2^{(k-j)\left(\alpha-\alpha_{2} / q\right) p}\right)\right\}^{1 / p} \\
+ & c\left\{\sum_{k=-\infty}^{\infty}\left(\sum_{j=k+2}^{\infty} 2^{j \alpha p}\left\|f_{j}\right\|_{L^{q}\left(\mathbb{R}^{n}\right)}^{p} 2^{(k-j)\left(\alpha-\alpha_{1} / q\right) p}\right)\right\}^{1 / p}
\end{aligned}
$$


$[5]$

$$
\begin{aligned}
= & c\left\{\sum_{j=-\infty}^{\infty} 2^{j \alpha p}\left\|f_{j}\right\|_{L^{q}\left(\mathbb{R}^{n}\right)}^{p}\left(\sum_{k=j-1}^{\infty} 2^{(k-j)\left(\alpha-\alpha_{2} / q\right) p}\right)\right\}^{1 / p} \\
& +c\left\{\sum_{j=-\infty}^{\infty} 2^{j \alpha p}\left\|f_{j}\right\|_{L^{q}\left(\mathbb{R}^{n}\right)}^{p}\left(\sum_{k=-\infty}^{j-2} 2^{(k-j)\left(\alpha-\alpha_{1} / q\right) p}\right)\right\}^{1 / p} \\
\leq & c\left\{\sum_{j=-\infty}^{\infty} 2^{j \alpha p}\left\|f_{j}\right\|_{L^{q}\left(\mathbb{R}^{n}\right)}^{p}\right\}^{1 / p} \\
= & c\|f\|_{\dot{K}_{q}^{\alpha . p}\left(\mathbb{R}^{n}\right)},
\end{aligned}
$$

since $\alpha-\alpha_{1} / q>0$ and $\alpha-\alpha_{2} / q<0$.

Now, let $1<p \leq \infty$. By Hölder's inequality, we then have

$$
\begin{aligned}
& \|T f\|_{\dot{K}_{q}^{\alpha . p}\left(\mathbb{R}^{n}\right)} \leq c\left\{\sum_{k=-\infty}^{\infty}\left(\sum_{j=-\infty}^{k+1} 2^{j \alpha p}\left\|f_{j}\right\|_{L^{q}\left(\mathbb{R}^{n}\right)}^{p} 2^{(k-j)\left(\alpha-\alpha_{2} / q\right) p / 2}\right)\right. \\
& \left.\times\left(\sum_{j=-\infty}^{k+1} 2^{(k-j)\left(\alpha-\alpha_{2} / q\right) p^{\prime} / 2}\right)^{p / p^{\prime}}\right\}^{1 / p} \\
& +c\left\{\sum_{k=-\infty}^{\infty}\left(\sum_{j=k+2}^{\infty} 2^{j \alpha p}\left\|f_{j}\right\|_{L^{q}\left(\mathbb{R}^{n}\right)}^{p} 2^{(k-j)\left(\alpha-\alpha_{2} / q\right) p / 2}\right)\right. \\
& \left.\times\left(\sum_{j=k+2}^{\infty} 2^{(k-j)\left(\alpha-\alpha_{1} / q\right) p^{\prime} / 2}\right)^{p / p^{\prime}}\right\}^{1 / p} \\
& \leq c\left\{\sum_{j=-\infty}^{\infty} 2^{j \alpha p}\left\|f_{j}\right\|_{L^{q}\left(\mathbb{R}^{n}\right)}^{p}\left(\sum_{k=j-1}^{\infty} 2^{(k-j)\left(\alpha-\alpha_{2} / q\right) p / 2}\right)\right\}^{1 / p} \\
& +c\left\{\sum_{j=-\infty}^{\infty} 2^{j \alpha p}\left\|f_{j}\right\|_{L^{q}\left(\mathbb{R}^{n}\right)}^{p}\left(\sum_{k=-\infty}^{j-2} 2^{(k-j)\left(\alpha-\alpha_{1} / q\right) p / 2}\right)\right\}^{1 / p} \\
& \leq c\left\{\sum_{j=-\infty}^{\infty} 2^{j \alpha p}\left\|f_{j}\right\|_{L^{a}\left(\mathbb{R}^{n}\right)}^{p}\right\}^{1 / p} \\
& =c\|f\|_{\dot{K}_{q}^{\alpha . p}\left(\mathbb{R}^{n}\right)} .
\end{aligned}
$$

This finishes the proof of Theorem 2.1.

Let $\Omega$ be a homogeneous function of degree zero on $\mathbb{R}^{n}$ and $\Omega \in L^{r}\left(S^{n-1}\right)$ for certain $r \in[1, \infty]$. The rough Hardy-Littlewood maximal function $M_{\Omega}$ is defined by

$$
M_{\Omega} f(x)=\sup _{t>0} \frac{1}{t^{n}} \int_{|y|<t}\left|\Omega\left(y^{\prime}\right) f(x-y)\right| d y,
$$


where $y^{\prime}=y|y|^{-1}$.

The following result for the boundedness of $M_{\Omega}$ on the Lebesgue spaces with power weights can be found in [5, p. 874].

LEMMA 2.1 ([5]). If $\Omega \in L^{r}\left(S^{n-1}\right), 1 \leq r \leq \infty, 1<q<\infty$ and

$$
\max \left(-n,-1-(n-1) q / r^{\prime}\right)<\beta<\min \left(n(q-1), q-1+(n-1) q / r^{\prime}\right),
$$

then $M_{\Omega}$ is bounded on $L_{|x|^{\beta}}^{q}\left(\mathbb{R}^{n}\right)$.

As a simple corollary of Theorem 2.1 and Lemma 2.1, we have the following corollary, which will be used in the proof of our main theorem.

COROLlaRY 2.1. Let $\Omega \in L^{r}\left(S^{n-1}\right), 1 \leq r \leq \infty, 1<q<\infty, 0<p \leq \infty$ and

$$
\max \left(-\frac{n}{q},-\frac{1}{q}-\frac{n-1}{r^{\prime}}\right)<\alpha<\min \left(n\left(1-\frac{1}{q}\right), 1-\frac{1}{q}+\frac{n-1}{r^{\prime}}\right),
$$

then $M_{\Omega}$ is bounded on $\dot{K}_{q}^{\alpha, p}\left(\mathbb{R}^{n}\right)$.

To prove one of our main theorems, we also need the following two lemmas. The first one can be found in [18, p. 251] and the second one in [8].

LEMMA 2.2 ([18]). Let $\Omega \in L^{r}\left(S^{n-1}\right)$ and $r \in[1, \infty]$. If $a>0,0<d \leq r$ and $-n+(n-1) d / r<\beta<\infty$, then

$$
\left(\int_{|x| \leq a|y|}|\Omega(x-y)|^{d}|x|^{\beta} d x\right)^{1 / d} \leq c|y|^{(\beta+n) / d}\|\Omega\|_{L^{r}\left(\mathbb{R}^{n}\right)} .
$$

In fact, when $1<r \leq \infty$, this lemma is just a special case of [18, Lemma 1]. The case $r=1$ is easy to prove using polar coordinates. We omit the details.

LeMma 2.3 ([8]). Let $\alpha \in \mathbb{R}$ and $1 \leq p, q<\infty$. Then $f \in \dot{K}_{q}^{\alpha, p}\left(\mathbb{R}^{n}\right)$ if and only if

$$
\left|\int_{\mathbb{R}^{n}} f(x) g(x) d x\right|<\infty
$$

for every $g \in \dot{K}_{q^{\prime}}^{-\alpha, p^{\prime}}\left(\mathbb{R}^{n}\right)$ and, in this case,

$$
\|f\|_{\dot{K}_{q}^{\alpha, p}\left(\mathbb{R}^{n}\right)}=\sup \left\{\left|\int_{\mathbb{R}^{n}} f(x) g(x) d x\right|:\|g\|_{\dot{K}_{q^{\prime}}^{-\alpha, p^{\prime}\left(\mathbb{R}^{n}\right)}} \leq 1\right\} .
$$

Now, we are in a position to state one of our main theorems. 
THEOREM 2.2. Let $\Omega \in L^{r}\left(S^{n-1}\right)$ for $r \in[1, \infty]$ and let $0<p \leq \infty, 1<q<\infty$ and $\max \left(-n / q,-1 / q-(n-1) / r^{\prime}\right)<\alpha<\min \left(n(1-1 / q), 1-1 / q+(n-1) / r^{\prime}\right)$. If a sublinear operator $T$ is bounded on $L^{q}\left(\mathbb{R}^{n}\right)$ and there is a constant $c$ independent off such that

$$
|T(f)(x)| \leq c \int_{\mathbb{R}^{n}} \frac{|\Omega(x-y)|}{|x-y|^{n}}|f(y)| d y
$$

for any $f \in L^{1}\left(\mathbb{R}^{n}\right)$ with compact support and $x \notin \operatorname{supp} f$, then $T$ is also bounded on $\dot{K}_{q}^{\alpha, p}\left(\mathbb{R}^{n}\right)$.

ProOF. Write

$$
f(x)=\sum_{k=-\infty}^{\infty} f(x) \chi_{k}(x) \equiv \sum_{k=-\infty}^{\infty} f_{k}(x)
$$

and

$$
\begin{aligned}
\|T f\|_{\dot{K}_{q}^{\alpha, p}\left(\mathbb{R}^{n}\right)} & \left\{\sum_{k=-\infty}^{\infty} 2^{k \alpha p}\left\|\chi_{k} T f\right\|_{L^{q}\left(\mathbb{R}^{n}\right)}^{p}\right\}^{1 / p} \\
\leq & c\left\{\sum_{k=-\infty}^{\infty} 2^{k \alpha p}\left\|\chi_{k} T\left(\sum_{j=-\infty}^{k-2} f_{j}\right)\right\|_{L^{q}\left(\mathbb{R}^{n}\right)}^{p}\right\}^{1 / p} \\
& +c\left\{\sum_{k=-\infty}^{\infty} 2^{k \alpha p}\left\|\chi_{k} T\left(\sum_{j=k-1}^{k+1} f_{j}\right)\right\|_{L^{q}\left(\mathbb{R}^{n}\right)}^{p}\right\}^{1 / p} \\
& +c\left\{\sum_{k=-\infty}^{\infty} 2^{k \alpha p}\left\|\chi_{k} T\left(\sum_{j=k+2}^{\infty} f_{j}\right)\right\|_{L^{q}\left(\mathbb{R}^{n}\right)}^{p}\right\}^{1 / p} \\
\equiv & D_{1}+D_{2}+D_{3} .
\end{aligned}
$$

For $D_{2}$, by the $L^{q}\left(\mathbb{R}^{n}\right)$-boundedness of $T$, we have

$$
D_{2} \leq c\left\{\sum_{k=-\infty}^{\infty} 2^{k \alpha p}\left\|\sum_{j=k-1}^{k+1} f_{j}\right\|_{L^{q}\left(\mathbb{R}^{n}\right)}^{p}\right\}^{1 / p} \leq c\|f\|_{K_{q}^{\alpha, p}\left(\mathbb{R}^{n}\right)} .
$$

For $D_{1}$, note that when $x \in A_{k}, j \leq k-2$, and $y \in A_{j}$, then $2|y| \leq|x|$. Therefore, for $x \in A_{k}$,

$$
\left|T\left(\sum_{j=-\infty}^{k-2} f_{j}\right)(x)\right| \leq c \int_{\mathbb{R}^{n}} \frac{|\Omega(x-y)|}{|x-y|^{n}}\left|\sum_{j=-\infty}^{k-2} f_{j}(y)\right| d y
$$




$$
\begin{aligned}
& \leq \frac{c}{|x|^{n}} \int_{|x-y| \leq 3|x| / 2}|\Omega(x-y)|\left|\sum_{j=-\infty}^{k-2} f_{j}(y)\right| d y \\
& \leq c M_{\Omega}\left(\sum_{j=-\infty}^{k-2} f_{j}\right)(x) .
\end{aligned}
$$

Thus, from Corollary 2.1 , it follows that

$$
\begin{aligned}
D_{1} & \leq c\left\{\sum_{k=-\infty}^{\infty} 2^{k \alpha p}\left\|\chi_{k} M_{\Omega}\left(\sum_{j=-\infty}^{k-2} f_{j}\right)\right\|_{L^{q}\left(\mathbb{R}^{n}\right)}^{p}\right\}^{1 / p} \\
& \leq c\left\|M_{\Omega}\left(\sum_{j=-\infty}^{k-2} f_{j}\right)\right\|_{\dot{K}_{q}^{\alpha, p}\left(\mathbb{R}^{n}\right)} \\
& \leq c\left\|\sum_{j=-\infty}^{k-2} f_{j}\right\|_{\dot{K}_{q}^{\alpha, p}\left(\mathbb{R}^{n}\right)} \\
& \leq c\|f\|_{\dot{K}_{q}^{\alpha . p}\left(\mathbb{R}^{n}\right)} .
\end{aligned}
$$

Now, let us turn to the estimate for $D_{3}$. We first assume $1<p<\infty$. Note that when $j \geq k+2, x \in A_{k}$, and $y \in A_{j}$, then $2|x| \leq|y|$ and

$$
\begin{aligned}
\left|T\left(\sum_{j=k+2}^{\infty} f_{j}\right)(x)\right| & \leq c \int_{\mathbb{R}^{n}} \frac{|\Omega(x-y)|}{|x-y|^{n}}\left|\sum_{j=k+2}^{\infty} f_{j}(y)\right| d y \\
& \leq c \int_{2|x| \leq|y|} \frac{|\Omega(x-y)|}{|y|^{n}}\left|\sum_{j=k+2}^{\infty} f_{j}(y)\right| d y \\
& \equiv c T_{0}\left(\left|\sum_{j=k+2}^{\infty} f_{j}\right|\right)(x) .
\end{aligned}
$$

Set

$$
T_{1}(g)(x)=\frac{1}{|x|^{n}} \int_{|y| \leq 2|x|}|\Omega(x-y)| g(y) d y .
$$

Then by Lemma 2.3 and Corollary 2.1 , when $1<p<\infty$, we obtain

$$
\begin{aligned}
D_{3} & \leq c\left\|T_{0}\left(\sum_{j=k+2}^{\infty} f_{j}\right)\right\|_{\dot{K}_{q}^{\alpha, p}\left(\mathbb{R}^{n}\right)} \\
& =c \sup _{\|g\|_{\substack{\dot{\alpha}_{q^{\prime}}^{-\alpha . p^{\prime}}\left(\mathbb{R}^{n}\right)}} \leq 1}\left|\left\langle T_{0}\left(\left|\sum_{j=k+2}^{\infty} f_{j}\right|\right), g\right\rangle\right|
\end{aligned}
$$




$$
\begin{aligned}
& =c \sup _{\|g\|_{\dot{K}_{q^{\prime}}^{-\alpha, p^{\prime}}\left(\mathbb{R}^{n}\right)} \leq 1}\left|\left\langle\left|\sum_{j=k+2}^{\infty} f_{j}\right|, T_{1}(g)\right\rangle\right| \\
& \leq c\left\|\sum_{j=k+2}^{\infty} f_{j}\right\|_{\dot{K}_{q}^{-\alpha, p}\left(\mathbb{R}^{n}\right)}\|g\|_{\dot{K}_{q^{\prime}}^{-\alpha, p^{\prime}}\left(\mathbb{R}^{n}\right)} \leq 1 \\
& \leq c\|f\|_{\dot{K}_{q}^{\alpha, p}\left(\mathbb{R}^{n}\right)} \sup _{\|g\|_{\dot{K}_{q^{\prime}}^{-\alpha, p^{\prime}}\left(\mathbb{R}^{n}\right)} \leq 1}\left\|M_{\Omega}(g)\right\|_{\dot{K}_{q^{\prime}}^{\alpha^{\alpha}, p^{\prime}}\left(\mathbb{R}^{n}\right)}(g) \|_{\dot{K}_{q^{\prime}}^{-\alpha, p^{\prime}}\left(\mathbb{R}^{n}\right)} \\
& \leq c\|f\|_{\dot{K}_{q}^{\alpha, p}\left(\mathbb{R}^{n}\right)} \sup _{\|g\|_{\dot{K}_{q^{\prime}}^{-\alpha, p^{\prime}}\left(\mathbb{R}^{n}\right)} \leq 1}\|g\|_{\dot{K}_{q^{\prime}}^{-\alpha, p^{\prime}}\left(\mathbb{R}^{n}\right)} \\
& \leq c\|f\| \|_{\dot{K}_{q}^{\alpha, p}\left(\mathbb{R}^{n}\right)},
\end{aligned}
$$

since $\max \left(-n / q^{\prime},-1 / q^{\prime}-(n-1) / r^{\prime}\right)<-\alpha<\min \left(n / q, 1 / q+(n-1) / r^{\prime}\right)$.

Now, let $0<p \leq 1$. In this case, we consider two sub-cases. First, suppose $1 \leq r^{\prime} \leq q$ and, therefore, $\alpha>-n / q$. When $x \in A_{k}$ and $j \geq k+2$, by Hölder's inequality, we have

$$
\begin{aligned}
\left|T\left(\sum_{j=k+2}^{\infty} f_{j}\right)(x)\right| & \leq c \sum_{j=k+2}^{\infty} \int_{\mathbb{R}^{n}} \frac{|\Omega(x-y)|}{|x-y|^{n}}\left|f_{j}(y)\right| d y \\
& \leq c \sum_{j=k+2}^{\infty} 2^{-j n / q}\|\Omega\|_{L^{q^{\prime}\left(S^{n-1}\right)}}\left\|f_{j}\right\|_{L^{q}\left(\mathbb{R}^{n}\right)} \\
& \leq c\|\Omega\|_{L^{r}\left(S^{n-1}\right)} \sum_{j=k+2}^{\infty} 2^{-j n / q}\left\|f_{j}\right\|_{L^{q}\left(\mathbb{R}^{n}\right)} .
\end{aligned}
$$

Thus we have

$$
\begin{aligned}
D_{3} & \leq c\left\{\sum_{k=-\infty}^{\infty} 2^{k(\alpha+n / q) p}\left(\sum_{j=k+2}^{\infty} 2^{-j n / q}\left\|f_{j}\right\|_{L^{q}\left(\mathbb{R}^{n}\right)}\right)^{p}\right\}^{1 / p} \\
& \leq c\left\{\sum_{j=-\infty}^{\infty} 2^{-j n p / q}\left\|f_{j}\right\|_{L^{q}\left(\mathbb{R}^{n}\right)}^{p}\left(\sum_{k=-\infty}^{j-2} 2^{k(\alpha+n / q) p}\right)\right\}^{1 / p} \\
& =c\left\{\sum_{j=-\infty}^{\infty} 2^{j \alpha p}\left\|f_{j}\right\|_{L^{q}\left(\mathbb{R}^{n}\right)}^{p}\right\}^{1 / p} \\
& \leq c\|f\|_{\dot{K}_{q}^{\alpha . p}\left(\mathbb{R}^{n}\right)} .
\end{aligned}
$$

Now suppose $r^{\prime}>q$ and, therefore, $\alpha>-1 / q-(n-1) / r^{\prime}$. Let $s=1-r+r / q=$ $1-r / q^{\prime}$. Then $(1-s) q^{\prime}=r$ and $s q=(1-r) q+r \leq r$. When $x \in A_{k}$ and $j \geq k+2$, 
by Hölder's inequality, we have

$$
\begin{aligned}
\left|T\left(\sum_{j=k+2}^{\infty} f_{j}\right)(x)\right| \leq & c \sum_{j=k+2}^{\infty} 2^{-j n / q}\|\Omega\|_{L^{\left(1-s q^{\prime}\left(S^{n-1}\right)\right.}}^{1-s} \\
& \times\left(\int_{\mathbb{R}^{n}}\left|f_{j}(y)\right|^{q}|\Omega(x-y)|^{s q} d y\right)^{1 / q} \\
\leq & c \sum_{j=k+2}^{\infty} 2^{-j n / q}\left(\int_{\mathbb{R}^{n}}\left|f_{j}(y)\right|^{q}|\Omega(x-y)|^{(1-r) q+r} d y\right)^{1 / q} .
\end{aligned}
$$

We choose $\beta \in \mathbb{R}$ and $0<\epsilon<1$ such that $\alpha>\beta / q>-1 / q-(n-1) / r^{\prime}$ and $\alpha-\beta / q>(1-\epsilon) n / q$. Then, by Hölder's inequality and Lemma 2.2, we obtain

$$
\begin{aligned}
\| \chi_{k} T & \left(\sum_{j=k+2}^{\infty} f_{j}\right) \|_{L^{q}\left(\mathbb{R}^{n}\right)} \\
\leq & c\left(\sum_{j=k+2}^{\infty} 2^{-j n(1-\epsilon) q^{\prime} / q}\right)^{1 / q^{\prime}} \\
& \times\left\|X_{k}\left[\sum_{j=k+2}^{\infty} 2^{-j n \epsilon}\left(\int_{\mathbb{R}^{n}}\left|f_{j}(y)\right|^{q}|\Omega(x-y)|^{(1-r) q+r} d y\right)\right]^{1 / q}\right\|_{L^{q}\left(\mathbb{R}^{n}\right)} \\
\leq & c 2^{-k n(1-\epsilon) / q}\left\{\sum_{j=k+2}^{\infty} 2^{-j n \epsilon} 2^{-k \beta} \int_{\mathbb{R}^{n}}\left|f_{j}(y)\right|^{q}\right. \\
& \left.\times\left(\int_{|x| \leq|y| / 2}|\Omega(x-y)|^{(1-r) q+r}|x|^{\beta} d x\right) d y\right\}^{1 / q} \\
\leq & c 2^{-k(n(1-\epsilon)+\beta) / q}\left\{\sum_{j=k+2}^{\infty} 2^{-j n \epsilon} \int_{\mathbb{R}^{n}}\left|f_{j}(y)\right|^{q}|y|^{\beta+n} d y\right\}^{1 / q} \\
\leq & c 2^{-k(n(1-\epsilon)+\beta) / q}\left\{\sum_{j=k+2}^{\infty} 2^{-j(n \epsilon-\beta-n) / q}\left\|f_{j}\right\|_{L^{q}\left(\mathbb{R}^{n}\right)}\right\} .
\end{aligned}
$$

Thus, in this case, we have

$$
\begin{aligned}
D_{3} & \leq c\left\{\sum_{k=-\infty}^{\infty} 2^{k(\alpha-(n(1-\epsilon)+\beta) / q) p}\left(\sum_{j=k+2}^{\infty} 2^{-j(n \epsilon-\beta-n) p / q}\left\|f_{j}\right\|_{L^{q}\left(\mathbb{R}^{n}\right)}^{p}\right)\right\}^{1 / p} \\
& =c\left\{\sum_{j=-\infty}^{\infty} 2^{-j(n \epsilon+\beta-n) p / q)}\left\|f_{j}\right\|_{L^{q}\left(\mathbb{R}^{n}\right)}^{p}\left(\sum_{k=-\infty}^{j-2} 2^{k(\alpha-(n(1-\epsilon)+\beta) / q) p}\right)\right\}^{1 / p}
\end{aligned}
$$




$$
\begin{aligned}
& =c\left\{\sum_{j=-\infty}^{\infty} 2^{j \alpha p}\left\|f_{j}\right\|_{L^{q}\left(\mathbb{R}^{n}\right)}^{p}\right\}^{1 / p} \\
& =c\|f\|_{\dot{K}_{a}^{\alpha . p}\left(\mathbb{R}^{n}\right)} .
\end{aligned}
$$

We leave the estimate for $D_{3}$ with $p=\infty$ to interested readers. This finishes the proof of Theorem 2.2 .

Note that $L_{|x|^{\alpha}}^{q}\left(\mathbb{R}^{n}\right)=\dot{K}_{q}^{\alpha / q, q}\left(\mathbb{R}^{n}\right)$. The following corollary is a consequence of Theorem 2.2.

COROLLARY 2.2. Let $\Omega \in L^{r}\left(S^{n-1}\right)$ and $r \in[1, \infty]$ and let $1<q<\infty$ and

$$
\max \left(-n,-1-\frac{(n-1) q}{r^{\prime}}\right)<\beta<\min \left(n(q-1), q-1+\frac{(n-1) q}{r^{\prime}}\right)
$$

If a sublinear operator $T$ satisfies (2.1) and is bounded on $L^{q}\left(\mathbb{R}^{n}\right)$, then $T$ is also bounded on $L_{|x|^{\beta}}^{q}\left(\mathbb{R}^{n}\right)$.

By examining the proof of Theorem 2.2, one can easily prove the following extension.

THEOREM 2.3. Let $0<p \leq \infty, 1 \leq r \leq \infty$, and

$$
\max \left(-\frac{n}{q},-\frac{1}{q}-\frac{n-1}{r^{\prime}}\right)<\alpha<\min \left(n\left(1-\frac{1}{q}\right), 1-\frac{1}{q}+\frac{n-1}{r^{\prime}}\right) .
$$

Suppose that $\Omega_{j} \in L^{r}\left(S^{n-1}\right)$ for $j \in I$ ( $I$ is some index set) and

$$
\left\|\Omega_{j}\right\|_{L^{\prime}\left(S^{n-1}\right)} \leq c_{0}<\infty
$$

where $c_{0}$ is independent of $j$. For sublinear operators $T_{j}$ 's, we define

$$
T^{*} f(x)=\sup _{j \in I}\left|T_{j}(f)(x)\right|
$$

If $T^{*}$ is bounded on $L^{q}\left(\mathbb{R}^{n}\right)$ and $T_{j}$ satisfies

$$
\left|T_{j}(f)(x)\right| \leq c_{0} \int_{\mathbb{R}^{n}} \frac{\left|\Omega_{j}(x-y)\right|}{|x-y|^{n}}|f(y)| d y
$$

for any $f \in L^{1}\left(\mathbb{R}^{n}\right)$ with compact support and $x \notin \operatorname{supp} f$, then $T^{*}$ is also bounded on $\dot{K}_{q}^{\alpha . p}\left(\mathbb{R}^{n}\right)$. 
COROLlaRY 2.3. Let $1<q<\infty, 1 \leq r \leq \infty$, and

$$
\max \left(-n,-1-(n-1) q / r^{\prime}\right)<\beta<\min \left(n(q-1), q-1+(n-1) q / r^{\prime}\right) .
$$

Suppose $\left\{\Omega_{j}\right\}_{j \in l}$ and $\left\{T_{j}\right\}_{j \in l}$ satisfy the conditions of Theorem 2.3. If $T^{*}$ is bounded on $L^{q}\left(\mathbb{R}^{n}\right)$, then $T^{*}$ is also bounded on $L_{|x|^{*}}^{q}\left(\mathbb{R}^{n}\right)$.

Now, let us turn to the fractional rough singular integrals. We first have the following theorem similar to Theorem 2.1.

THEOREM 2.4. Let $0<l<n$ and $T_{l}$ be a sublinear operator. If $T_{l}$ is bounded from $L_{|x|^{\alpha_{1}}}^{q_{q_{1}}}\left(\mathbb{R}^{n}\right)$ into $L_{\left.|x|\right|^{\mid q_{2}}}^{q_{2}}\left(\mathbb{R}^{n}\right)$ for all $\alpha \in\left(\alpha_{1}, \alpha_{2}\right)$ and some $q_{1}, q_{2} \in(1, \infty)$, where $\alpha_{1}, \alpha_{2} \in \mathbb{R}$ and $1 / q_{2}=1 / q_{1}-l / n$, then $T_{1}$ is also bounded from $\dot{K}_{q_{1}}^{\alpha_{1} p_{1}}\left(\mathbb{R}^{n}\right)$ into $\dot{K}_{q_{2}}^{\alpha, p_{2}}\left(\mathbb{R}^{n}\right)$ provided that $\alpha \in\left(\alpha_{1}, \alpha_{2}\right)$ and $0<p_{1} \leq p_{2} \leq \infty$.

ProOF. Note that if $p_{1}<p_{2}$, then $\dot{K}_{q_{2}}^{\alpha \cdot p_{1}}\left(\mathbb{R}^{n}\right) \subset \dot{K}_{q_{2}}^{\alpha . p_{2}}\left(\mathbb{R}^{n}\right)$. We only need to prove the theorem in the case $p_{1}=p_{2}$. Choose $\beta_{1}, \beta_{2} \in \mathbb{R}$ such that $\alpha_{1}<\beta_{1}<\alpha<\beta_{2}<$ $\alpha_{2}$. Write

$$
f(x)=\sum_{k=-\infty}^{\infty} f(x) \chi_{k}(x) \equiv \sum_{k=-\infty}^{\infty} f_{k}(x),
$$

it follows that

$$
\begin{aligned}
& \left\|T_{l} f\right\|_{\dot{K}_{q_{2}}^{\sigma, r_{1}\left(\mathbb{R}^{n}\right)}}=\left\{\sum_{k=-\infty}^{\infty} 2^{k \alpha p_{1}}\left\|\chi_{k} T_{l} f\right\|_{L^{q_{2}}\left(\mathbb{R}^{n}\right)}^{p_{1}}\right\}^{1 / p_{1}} \\
& \leq c\left\{\sum_{k=-\infty}^{\infty} 2^{k \alpha p_{1}}\left(\sum_{j=-\infty}^{k+1}\left\|\chi_{k} T_{l} f_{j}\right\|_{L^{q_{2}\left(\mathbb{R}^{n}\right)}}\right)^{p_{1}}\right\}^{1 / p_{1}} \\
& +c\left\{\sum_{k=-\infty}^{\infty} 2^{k \alpha p_{1}}\left(\sum_{j=k+2}^{\infty}\left\|\chi_{k} T_{l} f_{j}\right\|_{L^{q_{2}}\left(\mathbb{R}^{n}\right)}\right)^{p_{1}}\right\}^{1 / p_{1}} \\
& \leq c\left\{\sum_{k=-\infty}^{\infty} 2^{k\left(\alpha-\beta_{2}\right) p_{1}}\left(\sum_{j=-\infty}^{k+1}\left\|\chi_{k} T_{i} f_{j}\right\|_{L_{1,1 / k_{2} \psi_{2}}\left(\mathbb{R}^{n}\right)}\right)^{p_{1}}\right\}^{1 / p_{1}} \\
& +c\left\{\sum_{k=-\infty}^{\infty} 2^{k\left(\alpha-\beta_{1}\right) p_{1}}\left(\sum_{j=k+2}^{\infty}\left\|X_{1} T_{1} f_{j}\right\|_{L_{1, \mu_{1} \mu_{2} q_{2}}\left(\mathbb{R}^{n}\right)}\right)^{p_{1}}\right\}^{1 / p_{1}} \\
& \leq c\left\{\sum_{k=-\infty}^{\infty} 2^{k\left(\alpha-\beta_{2}\right) p_{1}}\left(\sum_{j=-\infty}^{k+1}\left\|f_{j}\right\|_{L_{1,1 \beta_{2} q_{1}}^{q_{1}}\left(\mathbb{R}^{n}\right)}\right)^{p_{1}}\right\}^{1 / p_{1}} \\
& +c\left\{\sum_{k=-\infty}^{\infty} 2^{k\left(\alpha-\beta_{1}\right) p_{1}}\left(\sum_{j=k+2}^{\infty}\left\|f_{j}\right\|_{L_{k, \mu_{1}}^{q_{1} \mu_{1}}\left(\mathbb{R}^{n}\right)}\right)^{p_{1}}\right\}^{1 / p_{1}}
\end{aligned}
$$




$$
\begin{aligned}
& \leq c\left\{\sum_{k=-\infty}^{\infty} 2^{k\left(\alpha-\beta_{2}\right) p_{1}}\left(\sum_{j=-\infty}^{k+1}\left\|f_{j}\right\|_{L^{q_{1}\left(\mathbb{R}^{n}\right)}} 2^{j \beta_{2}}\right)^{p_{1}}\right\}^{1 / p_{1}} \\
& +c\left\{\sum_{k=-\infty}^{\infty} 2^{k\left(\alpha-\beta_{1}\right) p_{1}}\left(\sum_{j=k+2}^{\infty}\left\|f_{j}\right\|_{L^{q_{1}\left(\mathbb{R}^{n}\right)}} 2^{j \beta_{1}}\right)^{p_{1}}\right\}^{1 / p_{1}} \\
& \leq c\left\{\sum_{k=-\infty}^{\infty}\left(\sum_{j=-\infty}^{k+1} 2^{j \alpha}\left\|f_{j}\right\|_{L^{q_{1}\left(\mathbb{R}^{n}\right)}} 2^{j\left(\beta_{2}-\alpha\right)}\right)^{p_{1}}\right\}^{1 / p_{1}} \\
& +c\left\{\sum_{k=-\infty}^{\infty}\left(\sum_{j=k+2}^{\infty} 2^{j \alpha}\left\|f_{j}\right\|_{L^{q_{1}\left(\mathbb{R}^{n}\right)}} 2^{j\left(\beta_{1}-\alpha\right)}\right)^{p_{1}}\right\}^{1 / p_{1}} \\
& \leq c\left\{\sum_{j=-\infty}^{\infty} 2^{j \alpha p_{1}}\left\|f_{j}\right\|_{L^{q_{1}\left(\mathbb{R}^{n}\right)}}^{p_{1}}\right\}^{1 / p_{1}} \\
& =c\|f\|_{K_{q_{1}}^{\alpha_{j} p_{1}\left(\mathbb{R}^{n}\right)},}
\end{aligned}
$$

where in the second-to-last inequality, we have employed the facts $\beta_{2}-\alpha>0$ and $\beta_{1}-\alpha<0$. This finishes the proof of Theorem 2.4.

The following lemma can be found in [18, p. 250].

LEMMA 2.4. Let $0<l<n, 1<q_{1}<n / l, 1 / q_{2}=1 / q_{1}-l / n, n /(n-l) \leq r \leq \infty$ and $\Omega \in L^{r}\left(S^{n-1}\right)$. If there is a constant $c$ independent of $f$ such that the sublinear operator $T_{l}$ satisfies that

$$
\left|T_{l}(f)(x)\right| \leq c \int_{\mathbb{R}^{n}} \frac{|\Omega(x-y)|}{|x-y|^{n-l}}|f(y)| d y
$$

for any $f \in L^{1}\left(\mathbb{R}^{n}\right)$ with compact support and $x \notin \operatorname{supp} f$, then $T_{l}$ is also bounded from $L_{|x| q_{1} \beta}^{q_{1}}\left(\mathbb{R}^{n}\right)$ into $L_{|x| q_{2} \beta}^{q_{2}}\left(\mathbb{R}^{n}\right)$ provided that

$$
l+\max \left(-\frac{n}{q_{1}},-\frac{1}{q_{1}}-\frac{n-1}{r^{\prime}}\right)<\beta<-l+\min \left(\frac{n}{q_{2}^{\prime}}, \frac{1}{q_{2}^{\prime}}+\frac{n-1}{r^{\prime}}\right) .
$$

As a simple corollary of Theorem 2.4 and Lemma 2.4, we have

COROLLARY 2.4. Assume that $0<l<n, 0<p_{1} \leq p_{2} \leq \infty, 1<q_{1}<n / l$, $1 / q_{2}=1 / q_{1}-l / n, n /(n-l) \leq r \leq \infty$ and $\Omega \in L^{r}\left(S^{n-1}\right)$. If a sublinear operator $T_{l}$ satisfies (2.4), then $T_{l}$ is also bounded from $\dot{K}_{q_{1}}^{\alpha, p_{1}}\left(\mathbb{R}^{n}\right)$ into $\dot{K}_{q_{2}}^{\alpha, p_{2}}\left(\mathbb{R}^{n}\right)$, provided that

$$
l+\max \left(-\frac{n}{q_{1}},-\frac{1}{q_{1}}-\frac{n-1}{r^{\prime}}\right)<\beta<-l+\min \left(\frac{n}{q_{2}^{\prime}}, \frac{1}{q_{2}^{\prime}}+\frac{n-1}{r^{\prime}}\right) .
$$


Now we come to consider the end-point cases of Theorems 2.1, 2.2 and 2.4. First, we will establish the boundedness of $M_{\Omega}$ on weak Herz spaces.

THEOREM 2.5. Let $\Omega \in L^{r}\left(S^{n-1}\right)$ and $r \in[1, \infty]$ and let $0<p \leq \infty$ and

$$
-n+(n-1) / r<\alpha<0 .
$$

If $r>1$, or $r=1$ and $M_{\Omega}$ is of weak type $(1,1)$, then $M_{\Omega}$ is type $\left(\dot{K}_{1}^{\alpha, p}\left(\mathbb{R}^{n}\right)\right.$, $\left.W \dot{K}_{1}^{\alpha, p}\left(\mathbb{R}^{n}\right)\right)$.

ProOF. Write

$$
f(x)=\sum_{j=-\infty}^{\infty} f(x) \chi_{j}(x) \equiv \sum_{j=-\infty}^{\infty} f_{j}(x)
$$

and

$$
\begin{aligned}
\lambda\left\{\sum_{k=-\infty}^{\infty} 2^{k \alpha p}\left|\left\{x \in A_{k}: M_{\Omega} f(x)>\lambda\right\}\right|^{p}\right\}^{1 / p} \\
\left.\leq c \lambda\left\{\sum_{k=-\infty}^{\infty} 2^{k \alpha p} \mid\left\{x \in A_{k}:\left|M_{\Omega}\left(\sum_{j=-\infty}^{k+1} f_{j}\right)(x)\right|>\lambda / 2\right\}\right\}^{p}\right\}^{1 / p} \\
\left.+c \lambda\left\{\sum_{k=-\infty}^{\infty} 2^{k \alpha p} \mid\left\{x \in A_{k}:\left|M_{\Omega}\left(\sum_{j=k+2}^{\infty} f_{j}\right)(x)\right|>\lambda / 2\right\}\right\}^{p}\right\}^{1 / p} \\
\equiv E_{1}+E_{2} .
\end{aligned}
$$

In [2], Christ and Rubio de Francia have proved that when $r>1, M_{\Omega}$ is of weak type $(1,1)$. By this and the hypothesis in the case $r=1$, for $E_{1}$, we have

$$
\begin{aligned}
E_{1} & \leq c\left\{\sum_{k=-\infty}^{\infty} 2^{k \alpha p}\left\|\sum_{j=-\infty}^{k+1} f_{j}\right\|_{L^{1}\left(\mathbb{R}^{n}\right)}^{p}\right\}^{1 / p} \\
& \leq c\left\{\sum_{k=-\infty}^{\infty} 2^{k \alpha p}\left(\sum_{j=-\infty}^{k+1}\left\|f_{j}\right\|_{L^{\prime}\left(\mathbb{R}^{n}\right)}\right)^{p}\right\}^{1 / p} .
\end{aligned}
$$

Note that $\alpha<0$. If $0<p \leq 1$, then

$$
E_{1} \leq c\left\{\sum_{j=-\infty}^{\infty}\left\|f_{j}\right\|_{L^{1}\left(\mathbb{R}^{n}\right)}^{p}\left(\sum_{k=j-1}^{\infty} 2^{k \alpha p}\right)\right\}^{1 / p}
$$




$$
\begin{aligned}
& =c\left\{\sum_{j=-\infty}^{\infty} 2^{j \alpha p}\left\|f_{j}\right\|_{L^{1}\left(\mathbb{R}^{n}\right)}^{p}\right\}^{1 / p} \\
& =c\|f\|_{\dot{K}_{1}^{\alpha . p}\left(\mathbb{R}^{n}\right)} .
\end{aligned}
$$

Now, let $1<p \leq \infty$. By Hölder's inequality, we then deduce

$$
\begin{aligned}
E_{1} & \leq c\left\{\sum_{k=-\infty}^{\infty} 2^{k \alpha p / 2}\left(\sum_{j=-\infty}^{k+1} 2^{j \alpha p / 2}\left\|f_{j}\right\|_{L^{\prime}\left(\mathbb{R}^{n}\right)}^{p}\right)\left(\sum_{j=-\infty}^{k+1} 2^{(k-j) \alpha p^{\prime} / 2}\right)^{p / p^{\prime}}\right\}^{1 / p} \\
& \leq c\left\{\sum_{j=-\infty}^{\infty} 2^{j \alpha p / 2}\left\|f_{j}\right\|_{L^{\prime}\left(\mathbb{R}^{n}\right)}^{p}\left(\sum_{j=k-1}^{\infty} 2^{k \alpha p / 2}\right)\right\}^{1 / p} \\
& \leq c\left\{\sum_{j=-\infty}^{\infty} 2^{j \alpha p}\left\|f_{j}\right\|_{L^{\prime}\left(\mathbb{R}^{n}\right)}^{p}\right\}^{1 / p} \\
& =c\|f\|_{\dot{K}_{1}^{\alpha, p}\left(\mathbb{R}^{n}\right)} .
\end{aligned}
$$

To estimate $E_{2}$, note that $-n+(n-1) / r<\alpha<0$. We can choose $\alpha_{1} \in \mathbb{R}$ such that $0<\alpha_{1}<\alpha+n-(n-1) / r$. When $j \geq k+2$, by Lemma 2.2, we have

$$
\begin{aligned}
\| \chi_{i} & M_{\Omega}\left(\sum_{j=k+2}^{\infty} f_{j}\right) \|_{L^{1}\left(\mathbb{R}^{n}\right)} \\
& \leq c \sum_{j=k+2}^{\infty} 2^{-j n} \int_{A_{k}}\left(\int_{A_{j}}|\Omega(x-y)|\left|f_{j}(y)\right| d y\right) d x \\
& \leq c \sum_{j=k+2}^{\infty} 2^{-j n+k\left(\alpha_{1}-\alpha\right)} \int_{A_{j}}\left|f_{j}(y)\right|\left(\int_{|x| \leq|y| / 2}|\Omega(x-y) \| x|^{\alpha_{1}-\alpha} d x\right) d y \\
& \leq c \sum_{j=k+2}^{\infty} 2^{-j n+k\left(\alpha_{1}-\alpha\right)} \int_{A_{j}}\left|f_{j}(y) \| y\right|^{\alpha-\alpha_{1}+n} d y \\
& \leq c \sum_{j=k+2}^{\infty} 2^{(k-j)\left(\alpha_{1}-\alpha\right)}\left\|f_{j}\right\|_{L^{1}\left(\mathbb{R}^{n}\right) .}
\end{aligned}
$$

Thus,

$$
\begin{aligned}
E_{2} & \leq c\left\{\sum_{k=-\infty}^{\infty} 2^{k \alpha p}\left\|\chi_{k} M_{\Omega}\left(\sum_{j=k+2}^{\infty} f_{j}\right)\right\|_{L^{1}\left(\mathbb{R}^{n}\right)}^{p}\right\}^{1 / p} \\
& \leq c\left\{\sum_{k=-\infty}^{\infty}\left(\sum_{j=k+2}^{\infty} 2^{j \alpha}\left\|f_{j}\right\|_{L^{1}\left(\mathbb{R}^{n}\right)} 2^{(k-j) \alpha_{1}}\right)^{p}\right\}^{1 / p}
\end{aligned}
$$


Note that $\alpha_{1}>0$. If $0<p \leq 1$, then

$$
\begin{aligned}
E_{2} & \leq c\left\{\sum_{j=-\infty}^{\infty} 2^{j \alpha p}\left\|f_{j}\right\|_{L^{\prime}\left(\mathbb{R}^{n}\right)}^{p}\left(\sum_{k=-\infty}^{j-2} 2^{(k-j) \alpha_{1} p}\right)\right\}^{1 / p} \\
& \leq c\left\{\sum_{j=-\infty}^{\infty} 2^{j \alpha p}\left\|f_{j}\right\|_{L^{\prime}\left(\mathbb{R}^{n}\right)}^{p}\right\}^{1 / p} \\
& =c\|f\|_{\dot{K}_{1}^{\alpha, p}\left(\mathbb{R}^{n}\right)} .
\end{aligned}
$$

If $1<p \leq \infty$, then by Hölder's inequality, we obtain

$$
\begin{aligned}
E_{2} & \leq c\left\{\sum_{k=-\infty}^{\infty}\left(\sum_{j=k+2}^{\infty} 2^{j \alpha p}\left\|f_{j}\right\|_{L^{\prime}\left(\mathbb{R}^{n}\right)}^{p} 2^{(k-j) \alpha_{1} p / 2}\right)\left(\sum_{j=k+2}^{\infty} 2^{(k-j) \alpha_{1} p^{\prime} / 2}\right)^{p / p^{\prime}}\right\}^{1 / p} \\
& \leq c\left\{\sum_{j=-\infty}^{\infty} 2^{j \alpha p}\left\|f_{j}\right\|_{L^{1}\left(\mathbb{R}^{n}\right)}^{p}\left(\sum_{k=-\infty}^{j-2} 2^{(k-j) \alpha_{1} p / 2}\right)\right\}^{1 / p} \\
& =c\left\{\sum_{j=-\infty}^{\infty} 2^{j \alpha p}\left\|f_{j}\right\|_{L^{1}\left(\mathbb{R}^{n}\right)}^{p}\right\}^{1 / p} \\
& =c\|f\|_{\dot{K}_{1}^{\alpha . p}\left(\mathbb{R}^{n}\right) .}
\end{aligned}
$$

This finishes the proof of Theorem 2.5.

By a proof similar to that of Theorem 2.5, one can prove the following

THEOREM 2.6. Suppose that $\Omega \in L^{r}\left(S^{n-1}\right)$ and $r \in[1, \infty]$ and let $0<p \leq \infty$ and $-n+(n-1) / r<\alpha<0$. If a sublinear operator $T$ is of weak type $(1,1)$ and satisfies (2.1), then $T$ is also bounded from $\dot{K}_{1}^{\alpha, p}\left(\mathbb{R}^{n}\right)$ into $W \dot{K}_{1}^{\alpha, p}\left(\mathbb{R}^{n}\right)$.

Note that $\dot{K}_{1}^{\alpha, 1}\left(\mathbb{R}^{n}\right)=L_{|x|^{\alpha}}^{1}\left(\mathbb{R}^{n}\right)$. Thus, Theorems 2.5 and 2.6 with the case $p=1$ are the same as [22, Proposition 8] with the power weights. In addition, Theorem 2.6 is also true for the maximal singular integrals. That is,

THEOREM 2.7. Let $1 \leq r \leq \infty, 0<p \leq \infty$ and $-n+(n-1) / r<\alpha<0$. Suppose $\left\{\Omega_{j}\right\}_{j \in I},\left\{T_{j}\right\}_{j \in I}$ and $T^{*}$ are the same as in Theorem 2.3 and satisfies (2.2) and (2.3). If $T^{*}$ is of weak type $(1,1)$, then $T^{*}$ maps continuously $\dot{K}_{1}^{\alpha, p}\left(\mathbb{R}^{n}\right)$ into $W \dot{K}_{1}^{\alpha, p}\left(\mathbb{R}^{n}\right)$.

For fractional singular integrals, we have some theorems similar to Theorems 2.6 and 2.7. 
THEOREM 2.8. Let $0<l<n, 0<p_{1} \leq p_{2} \leq \infty, q_{1}=n /(n-l) \leq r \leq \infty$,

$$
l-n+(n-1) / r<\alpha<0,
$$

and $\Omega \in L^{r}\left(S^{n-1}\right)$. If a sublinear operator $T_{1}$ satisfies (2.4) and is of weak type $\left(1, q_{1}\right)$, then $T_{1}$ is also type $\left(\dot{K}_{1}^{\alpha, p_{1}}\left(\mathbb{R}^{n}\right), W \dot{K}_{q_{2}}^{\alpha, p_{2}}\left(\mathbb{R}^{n}\right)\right)$.

PROOF. Note that if $p_{1}<p_{2}$, then $W \dot{K}_{q_{1}}^{\alpha, p_{1}}\left(\mathbb{R}^{n}\right) \subset W \dot{K}_{q_{1}}^{\alpha, p_{2}}\left(\mathbb{R}^{n}\right)$. We only need to prove the theorem in the case $p_{1}=p_{2}$. Write

$$
f(x)=\sum_{j=-\infty}^{\infty} f(x) \chi_{j}(x) \equiv \sum_{j=-\infty}^{\infty} f_{j}(x)
$$

and

$$
\begin{aligned}
& \lambda\left\{\sum_{k=-\infty}^{\infty} 2^{k \alpha p_{1}}\left|\left\{x \in A_{k}:\left|T_{l}(f)(x)\right|>\lambda\right\}\right|^{p_{1} / q_{1}}\right\}^{1 / p_{1}} \\
& \leq c \lambda\left\{\sum_{k=-\infty}^{\infty} 2^{k \alpha p_{1}}\left|\left\{x \in A_{k}:\left|T_{l}\left(\sum_{j=-\infty}^{k+1} f_{j}\right)(x)\right|>\lambda / 2\right\}\right|^{p_{1} / q_{1}}\right\}^{1 / p_{1}} \\
& \quad+c \lambda\left\{\sum_{k=-\infty}^{\infty} 2^{k \alpha p_{1}}\left|\left\{x \in A_{k}:\left|T_{i}\left(\sum_{j=k+2}^{\infty} f_{j}\right)(x)\right|>\lambda / 2\right\}\right|^{p_{1} / q_{1}}\right\}^{1 / p_{1}} \\
& \equiv F_{1}+F_{2} .
\end{aligned}
$$

Since $T_{l}$ is of weak type $\left(1, q_{1}\right)$, for $F_{1}$, we then have

$$
\begin{aligned}
F_{1} & \leq c\left\{\sum_{k=-\infty}^{\infty} 2^{k \alpha p_{1}}\left\|\sum_{j=-\infty}^{k+1} f_{j}\right\|_{L^{\prime}\left(\mathbb{R}^{n}\right)}^{p_{1}}\right\}^{1 / p_{1}} \\
& \leq c\left\{\sum_{k=-\infty}^{\infty}\left(\sum_{j=-\infty}^{k+1} 2^{j \alpha}\left\|f_{j}\right\|_{L^{\prime}\left(\mathbb{R}^{n}\right)} 2^{(k-j) \alpha}\right)^{p_{1}}\right\}^{1 / p_{1}} \\
& \leq c\left\{\sum_{j=-\infty}^{\infty} 2^{j \alpha p_{1}}\left\|f_{j}\right\|_{L^{\prime}\left(\mathbb{R}^{n}\right)}^{p_{1}}\right\}^{1 / p_{1}} \\
& =c\|f\|_{\dot{K}_{1}^{\alpha, p_{1}}\left(\mathbb{R}^{n}\right)},
\end{aligned}
$$

where we have used that $\alpha<0$ and some similar estimates used for the estimate of $E_{1}$. For the estimate of $F_{2}$, note that $l-n+(n-1) / r<\alpha<0$. We can choose $\beta_{1} \in \mathbb{R}$ such that

$$
0<\beta_{1}<\alpha+n-(n-1) / r-l
$$


When $j \geq k+1$, by Lemma 2.2 , we have

$$
\begin{aligned}
\| x_{k} T_{l} & \left(\sum_{j=k+2}^{\infty} f_{j}\right) \|_{L^{q_{1}\left(\mathbb{R}^{n}\right)}}\left[\int_{A_{k}}\left(\int_{A_{j}}\left|\Omega(x-y) \| f_{j}(y)\right| d y\right)^{q_{1}} d x\right]^{1 / q_{1}} \\
& \leq c \sum_{j=k+2}^{\infty} 2^{j(n-l)}\left[\int_{A_{j}}\left|f_{j}(y)\right|\left(\int_{A_{k}}|\Omega(x-y)|^{q_{1}}|x|^{\beta} d x\right)^{1 / q_{1}} d y\right. \\
& \leq c \sum_{j=k+2}^{\infty} 2^{-j(n-l)-k \beta / q_{1}} \int_{A_{j}}\left|f_{j}(y) \| y\right|^{(\beta+n) / q_{1}} d y \\
& \leq c \sum_{j=k+2}^{\infty} 2^{-j(n-l)-k \beta / q_{1}} \int_{j=k+2}^{\infty} 2^{(j-k) \beta / q_{1}}\left\|f_{j}\right\|_{L^{1}\left(\mathbb{R}^{n}\right)},
\end{aligned}
$$

where we choose $\beta \in \mathbb{R}$ such that $\alpha>\beta / q_{1}>-n / q_{1}+(n-1) / r=(n-1) / r-n+l$. Since $\alpha>(n-1) / r-n+l$, this is possible. Therefore,

$$
\begin{aligned}
F_{2} & \leq c\left\{\sum_{k=-\infty}^{\infty} 2^{k \alpha p_{1}}\left\|\chi_{k} T_{l}\left(\sum_{j=k+2}^{\infty} f_{j}\right)\right\|_{L^{q_{2}\left(\mathbb{R}^{n}\right)}}^{p_{1}}\right\}^{1 / p_{1}} \\
& \leq c\left\{\sum_{k=-\infty}^{\infty}\left(\sum_{j=k+2}^{\infty} 2^{j \alpha}\left\|f_{j}\right\|_{L^{1}\left(\mathbb{R}^{n}\right)} 2^{(j-k)\left(\beta q_{2}-\alpha\right)}\right)^{p_{1}}\right\}^{1 / p_{1}} \\
& \leq c\left\{\sum_{j=-\infty}^{\infty} 2^{j \alpha p_{1}}\left\|f_{j}\right\|_{L^{1}\left(\mathbb{R}^{n}\right)}^{p_{1}}\right\}^{1 / p_{1}} \\
& =c\|f\|_{\dot{K}_{1}^{\alpha, p_{1}}\left(\mathbb{R}^{n}\right)}
\end{aligned}
$$

by some similar estimates to those used for $E_{2}$. This finishes the proof of Theorem 2.8 .

If we take $p_{1}=1$ and $p_{2}=q_{1}$ in Theorem 2.8 , we then obtain

COROLlaRY 2.5. Let $0<l<n, q_{1}=n /(n-l) \leq r \leq \infty,-n+(n-1) / r<\alpha<0$ and $\Omega \in L^{r}\left(S^{n-1}\right)$. If a sublinear operator $T_{l}$ satisfies $(2.4)$ and is of weak type $\left(1, q_{1}\right)$, then $T_{l}$ is also of type $\left(L_{|x|^{\alpha}}\left(\mathbb{R}^{n}\right), W L_{|x|^{a t q_{1}}}^{q_{1}}\left(\mathbb{R}^{n}\right)\right)$.

We remark that Corollary 2.5 is the end-point case of [18, Theorem 2]. 


\section{Some applications}

In this section, we will give some applications of the theorems in Section 2. Let us begin with the following singular integral operators of Fefferman [7]:

$$
T_{h} f(x)=\text { p.v. } \int_{\mathbb{R}^{n}} h(x-y) \frac{\Omega(x-y)}{|x-y|^{n}} f(y) d y,
$$

where $h$ is a bounded radial function, $\Omega$ is homogeneous of degree 0 and belongs to $L^{1}\left(S^{n-1}\right)$. A result of Fan-Pan [6] states that if $\Omega \in H^{1}\left(S^{n-1}\right)$, the Hardy space on $S^{n-1}$ (see [3] or [6] for the definition), then this operator is bounded on $L^{q}\left(\mathbb{R}^{n}\right)$ for all $q \in(1, \infty)$. As a consequence of this result and Theorem 2.2, we have

COROLlary 3.1. Let $0<p \leq \infty, 1<q<\infty$ and $-1 / q<\alpha<1-1 / q$. Suppose $T$ is defined as in (3.1). If $h$ is a bounded radial function and $\Omega \in H^{1}\left(S^{n-1}\right)$, then $T_{h}$ is bounded on $\dot{K}_{q}^{\alpha, p}\left(\mathbb{R}^{n}\right)$.

From [18, Theorem 1] and Theorem 2.1 or [18, Theorem 1] with $\alpha=0$ and Theorem 2.3, we deduce the following.

COROLLARY 3.2. Suppose that when $r \in(1, \infty]$,

$$
\Omega \in L^{r}\left(S^{n-1}\right)
$$

or when $r=1, \Omega \in L \log ^{+} L\left(S^{n-1}\right)$. If $0<p \leq \infty, 1<q<\infty$,

$$
\max \left(-\frac{n}{q},-\frac{1}{q}-\frac{n-1}{r^{\prime}}\right)<\alpha<\min \left(n\left(1-\frac{1}{q}\right), 1-\frac{1}{q}+\frac{n-1}{r^{\prime}}\right)
$$

and $\Omega$ has mean value 0 on $S^{n-1}$, then

$$
T_{1}^{*} f(x)=\sup _{\epsilon>0}\left|\int_{|y|>\epsilon} \frac{\Omega(y)}{|y|^{n}} f(x-y) d y\right|
$$

is bounded on $\dot{K}_{q}^{\alpha, p}\left(\mathbb{R}^{n}\right)$.

We point out that the conditions of [18, Theorem 1] require

$$
\Omega \in L \log ^{+} L\left(S^{n-1}\right)
$$

when $r=1$.

When $q=p$, Corollary 3.2 is just [18, Theorem 1] and is sharp in this case; see [18]. 
Now, let us consider the following commutators of high order defined by

$$
T_{b, l}^{m} f(x)=\text { p.v. } \int_{\mathbb{R}^{n}}(b(x)-b(y))^{m} \frac{\Omega(x-y)}{|x-y|^{n-l}} f(y) d y,
$$

where $0 \leq l<\infty, b \in \operatorname{BMO}\left(\mathbb{R}^{n}\right)$ and $m \in \mathbb{N}$.

From [11, Theorem 1], [4, Theorem 4.3], Theorems 2.1 and 2.4, we deduce

COROllary 3.3. Let $0<p_{1} \leq p_{2} \leq \infty, 0 \leq l<n, 1<q_{1}<n / l, 1 / q_{2}=$ $1 / q_{1}-l / n, 1-1 / r>1 / q_{1}>l / n$ and $-n / q_{1}+l<\alpha<n\left(1-1 / r-1 / q_{1}\right)$. If $\Omega \in L^{r}\left(S^{n-1}\right), b \in \mathrm{BMO}\left(\mathbb{R}^{n}\right)$ and $m \in \mathbb{N}$, then $T_{b, l}^{m}$ in (3.2) is bounded from $\dot{K}_{q_{1}}^{\alpha, p_{1}}\left(\mathbb{R}^{n}\right)$ into $\dot{K}_{q_{2}}^{\alpha, p_{2}}\left(\mathbb{R}^{n}\right)$.

We now turn to the boundedness of $T_{1}$ in (3.1) on weak type Herz spaces. By the proposition in [21] and Theorem 2.6, we obtain

COROLLARY 3.4. Let $0<p \leq \infty$ and $-1<\alpha<0$. If $\Omega \in L \log ^{+} L\left(S^{n-1}\right)$ and has mean value zero, then $T_{1}$ in (3.1) is bounded from $\dot{K}_{1}^{\alpha, p}\left(\mathbb{R}^{n}\right)$ into $W \dot{K}_{1}^{\alpha, p}\left(\mathbb{R}^{n}\right)$.

Similarly, for the following fractional singular integrals $T^{l}$ defined by

$$
T^{l} f(x)=\text { p.v. } \int_{\mathbb{R}^{n}} \frac{\Omega(y)}{|y|^{n-l}} f(x-y) d y,
$$

we have the following corollary.

COROLlary 3.5. Let $0<p_{1} \leq p_{2} \leq \infty, 0<l<n, q_{1}=n /(n-l)<r \leq \infty$ and $l-n+(n-1) / r<\alpha<0$. If $\Omega \in L^{r}\left(S^{n-1}\right)$, then the operator $T^{l}$ in (3.3) maps continuously $\dot{K}_{1}^{\alpha, p_{1}}\left(\mathbb{R}^{n}\right)$ into $W \dot{K}_{q_{1}}^{\alpha, p_{2}}\left(\mathbb{R}^{n}\right)$.

In [4], Ding proved that if $\Omega \in L^{r}\left(S^{n-1}\right)$ and $q_{1}=n /(n-l)<r \leq \infty$, then the operator $T^{l}$ in (3.1) is of type $\left(1, q_{1}\right)$. This result and Theorem 2.8 imply Corollary 3.5.

Now let us turn to the boundedness of oscillatory singular integral operators defined by

$$
T f(x)=\text { p.v. } \int_{\mathbb{R}^{n}} e^{i P(x, y)} H(x-y) f(y) d y,
$$

where $P(x, y)$ is a real polynomial on $\mathbb{R}^{n} \times \mathbb{R}^{n}$, and $H(x)=\Omega(x)|x|^{-n}$ for all $|x| \neq 0$, and $\Omega$ is homogeneous of degree zero. If $H$ is a standard Calderón-Zygmund operator, the operator $T$ in (3.4) was introduced by Ricci-Stein in [20]. Jiang and Lu in [13] proved that if $\Omega \in L \log ^{+} L\left(S^{n-1}\right)$ and $\Omega$ has mean value 0 on $S^{n-1}$, then $T$ in (3.4) is bounded on $L^{q}\left(\mathbb{R}^{n}\right)$ for $q \in(1, \infty)$. The following corollary is a simple application of Theorem 2.2. 
COROLlaRY 3.6. Let $0<p \leq \infty, 1<q<\infty$ and $-1 / q<\alpha<1-1 / q$. If $H(x)=\Omega(x)|x|^{-n}, \Omega \in L \log ^{+} L\left(S^{n-1}\right)$ and $\Omega$ has mean value 0 on $S^{n-1}$, then $T$ in (3.4) is bounded on $\dot{K}_{q}^{\alpha, p}\left(\mathbb{R}^{n}\right)$ with the operator norm independent of the coefficients of $P$.

Chanillo and Christ in [1] showed that if $H$ is a standard Calderón-Zygmund kernel, then the operator $T$ in (3.4) is of weak type $(1,1)$ with the operator norm independent of the coefficients of $P$. Combining this with Theorem 2.6, we have

COROLlARY 3.7. Let $0<p \leq \infty$ and $-n<\alpha<0$. If $H$ is a standard Calderón-Zygmund kernel, then the operator $T$ in (3.4) is bounded from $\dot{K}_{1}^{\alpha, p}\left(\mathbb{R}^{n}\right)$ into $W \dot{K}_{1}^{\alpha, p}\left(\mathbb{R}^{n}\right)$ with the operator norm independent of the coefficients of $P$.

Pan in [19] also studied the uniform weak $(1,1)$-boundedness of the following oscillatory singular integral operators defined by

$$
T_{\lambda} f(x)=\text { p.v. } \int_{\mathbb{R}^{n}} e^{i \lambda \Phi(x, y)} H(x-y) \varphi(x, y) f(y) d y,
$$

where $\lambda \in \mathbb{R}, \varphi \in C_{0}^{\infty}\left(\mathbb{R}^{n} \times \mathbb{R}^{n}\right), \Phi$ is a real-valued smooth function on $\mathbb{R}^{n} \times \mathbb{R}^{n}$ and $H$ is a Calderón-Zygmund kernel. By Pan's Theorem 4 in [19] and Theorem 2.6, we obtain

COROLlaRY 3.8. Let $0<p \leq \infty,-n<\alpha<0$ and $H$ be a Calderón-Zygmund kernel. If $\Phi(x, y)=\phi(x-y)$ with $\phi \in C^{\infty}\left(\mathbb{R}^{n}\right)$ and there exists an $\alpha \in \mathbb{N}^{n}$ with $|\alpha|>1$ such that $\partial^{\alpha} \phi(0) / \partial x^{\alpha} \neq 0$, then the operator $T_{\lambda}$ in (3.5) is uniformly bounded in $\lambda \in \mathbb{R}$ from $\dot{K}_{1}^{\alpha, p}\left(\mathbb{R}^{n}\right)$ into $W \dot{K}_{1}^{\alpha, p}\left(\mathbb{R}^{n}\right)$.

Before we end this section, let us remark that more applications can be given to the (fractional) Hardy-Littlewood maximal operators related to $T_{1}$ in (3.1) and $T^{l}$ in (3.3), the two-dimensional Marcinkiewicz integrals and the commutators of the oscillatory singular integral operators with $\mathrm{BMO}\left(\mathbb{R}^{n}\right)$ functions; see $[10,21]$ and [4]. We omit the details.

\section{Acknowledgment}

The authors wish to express their deep thanks to the referee for many valuable comments. 


\section{References}

[1] S. Chanillo and M. Christ, 'Weak $(1,1)$ bounds for oscillatory singular integrals', Duke Math. J. 55 (1987), 141-155.

[2] M. Christ and J. L. Rubio de Francia, 'Weak type $(1,1)$ bounds for rough operators, II', Invent. Math. 93 (1988), 225-237.

[3] R. R. Coifman and G. Weiss, 'Extensions of Hardy spaces and their use in analysis', Bull. Amer. Math. Soc. 83 (1977), 569-645.

[4] Y. Ding, Some problems on oscillatory integral operators and fractional integral operators with rough kernels (Ph.D. Thesis, Beijing Normal University, Beijing, 1995).

[5] J. Duoandikoetxea, 'Weighted norm inequalities for homogeneous singular integrals', Trans. Amer. Math. Soc. 336 (1993), 869-880.

[6] D. Fan and Y. Pan, 'Singular integral operators with rough kernels supported by subvarieties', Amer. J. Math. 119 (1997), 799-839.

[7] R. Fefferman, 'A note on singular integrals', Proc. Amer. Math. Soc. 74 (1979), 266-270.

[8] E. Hernández and D. Yang, 'Interpolation of Herz spaces and applications', Math. Nachr. (to appear).

[9] C. Herz, 'Lipschitz spaces and Bernstein's theorem on absolutely convergent Fourier transforms', J. Math. Mech. 18 (1967), 283-324.

[10] G. Hu, 'A note on sublinear operators and power weights', J. Beijing Normal Univ. (Natur. Sci.) 31 (1995), 170-174.

[11] - 'Weighted norm inequalities for commutators of homogeneous singular integrals', Acta Math. Sinica (New Ser.) (Special Issue) 11 (1995), 77-88.

[12] G. Hu, S. Lu and D. Yang, 'The weak Herz spaces', J. Beijing Normal Univ. (Natur. Sci.) 33 (1997), 27-34.

[13] Y. Jiang and S. Lu, 'Oscillatory singular integrals with rough kernel', in: Harmonic Analysis in China (eds. M. Cheng, D. Deng, S. Gong and C. Yang), Mathematics and its Applications, vol. 327 (Kluwer Acad. Publ., London, 1995) pp. 135-145.

[14] X. Li and D. Yang, 'Boundedness of some sublinear operators on Herz spaces', Illinois J. Math. 40 (1996), 484-501.

[15] W. Liu and S. Lu, 'Rough operators on the weighted Herz spaces', J. Beijing Normal Univ. (Natur. Sci.) 31 (1995), 446-451.

[16] S. Lu, 'An extension of Soria-Weiss' theorem on sublinear operators and power weights', J. Beijing Normal Univ. (Natur. Sci.) 30 (1994), 313-316.

[17] S. Lu and D. Yang, 'Hardy-Littlewood-Sobolev theorem of fractional integration on Herz-type spaces and its applications', Canad. J. Math. 48 (1996), 363-380.

[18] B. Muckenhoupt and R. L. Wheeden, 'Weighted norm inequalities for singular and fractional integrals', Trans. Amer. Math. Soc. 161 (1971), 249-258.

[19] Y. Pan, 'Uniform weak (1, 1) bounds for oscillatory singular integrals', in: Harmonic Analysis in China (eds. M. Cheng, D. Deng, S. Gong and C. Yang), Mathematics and its Applications, vol. 327 (Kluwer Acad. Publ., London, 1995) pp. 210-219.

[20] F. Ricci and E. M. Stein, 'Harmonic analysis on nilpotent groups and singular integral, I', J. Funct. Anal. 73 (1987), 179-194.

[21] A. Seeger, 'Singular integral operators with rough convolution kernels', J. Amer. Math. Soc. 9 (1996), 95-105.

[22] F. Soria and G. Weiss, 'A remark on singular integrals and power weights', Indiana Univ. Math. J. 43 (1994), 187-204.

[23] E. M. Stein, 'Note on singular integrals', Proc. Amer. Math. Soc. 8 (1957), 250-254. 
Department of Applied Mathematics Institute of Information Engineering

Box 1001-47, Zhengzhou 450002

P. R. China
Department of Mathematics Beijing Normal University Beijing 100875 P. R. China e-mail: lusz@bnu.edu.cn e-mail: dcyang@bnu.edu.cn 